\title{
A blind deconvolution approach to high-resolution mapping of transcription factor binding sites from ChIP-seq data Desmond S Lun*, Ashley Sherrid ${ }^{\dagger \ddagger}$, Brian Weiner ${ }^{\S}$, David R Sherman ${ }^{\dagger}$ and James E Galagan ${ }^{\S}$
}

\begin{abstract}
Addresses: *Phenomics and Bioinformatics Research Centre, School of Mathematics and Statistics, and Australian Centre for Plant Functional Genomics, University of South Australia, Mawson Lakes Boulevard, Mawson Lakes, SA 5095, Australia. `Seattle Biomedical Research Institute, 307 Westlake Avenue North, Suite 500, Seattle, WA 98109, USA. ₹Molecular and Cellular Biology Graduate Program, University of Washington, Seattle, WA 98195, USA. \$Broad Institute of MIT and Harvard, 7 Cambridge Center, Cambridge, MA 02142, USA. ^Department of Global Health, University of Washington, Seattle, WA 98195, USA. ${ }^{\circledR}$ Department of Biomedical Engineering and Department of Microbiology, Boston University, 44 Cummington Street, Boston, MA 02215, USA.
\end{abstract}

Correspondence: Desmond S Lun. Email: desmond.lun@unisa.edu.au

Published: 22 December 2009

Genome Biology 2009, 10:R I42 (doi:10.1 I86/gb-2009-10-12-r|42)

The electronic version of this article is the complete one and can be found online at http://genomebiology.com/2009//0/I2/R/42
Received: 12 September 2009

Revised:

I5 November 2009

Accepted: 22 December 2009

๑ 2009 Lun et al.; licensee Biomed Central Ltd.

This is an open access article distributed under the terms of the Creative Commons Attribution license (http://creativecommons.org/licenses/ by/2.0), which permits unrestricted use, distribution, and reproduction in any medium, provided the original work is properly cited.

\begin{abstract}
We present CSDeconv, a computational method that determines locations of transcription factor binding from ChIP-seq data. CSDeconv differs from prior methods in that it uses a blind deconvolution approach that allows closely-spaced binding sites to be called accurately. We apply CSDeconv to novel ChIP-seq data for DosR binding in Mycobacterium tuberculosis and to existing data for GABP in humans and show that it can discriminate binding sites separated by as few as 40 bp.
\end{abstract}

\section{Background}

With the rapidly decreasing cost of DNA sequencing, chromatin immunoprecipitation (ChIP) followed by sequencing of the resulting DNA fragments (ChIP-seq) is fast becoming the most attractive method for the study of genome-wide protein-DNA interaction, yielding advantages such as lower cost, higher resolution, and a lower requirement for input material over the principal alternative, ChIP-chip, which involves hybridization of the immunoprecipitated fragments to a genomic microarray [1-3]. But to harness fully the potential of ChIP-seq, analysis techniques that accurately translate sequencing reads into reliable calls of the genomic locations of the sites of protein-DNA interaction are necessary. To date, a number of such analysis techniques have been developed [2,4-14]. These methods, however, generally do not identify distinct binding sites lying close together (separated by a distance on the order of $100 \mathrm{bp}$ or less), instead interpreting such cases as a single, incorrectly located binding site. Such cases of closely spaced binding sites arise regularly, especially in prokaryotic genomes (see, for example, $[15,16])$, and an analysis technique capable of making the correct calls is necessary for the full potential of ChIP-seq to be realized.

We present CSDeconv, a computational method that accurately identifies binding sites, including closely spaced binding sites, from ChIP-seq data. In contrast to 
prior methods that identify binding sites by searching for enrichment peaks in sequenced reads, we recognize that peaks cannot be clearly and distinctly resolved when binding sites are separated by short distances, and we therefore instead use a blind deconvolution approach in which we simultaneously estimate the shape of an enrichment peak as well as the location and magnitude of binding sites. Our work builds on many of the innovations introduced by Valouev and colleagues [4] to the analysis of ChIP-seq data in their method QuEST, including using kernel density estimation $[17,18]$ to estimate the probability density function associated with the location of sequencing reads.

To demonstrate the capabilities of CSDeconv, we have applied it to novel ChIP-seq data for the DosR (dormancy survival regulator) transcription factor in Mycobacterium tuberculosis (MTB) and to existing data collected by Valouev and colleagues [4] for the GABP (growth-associated binding protein) transcription factor in humans. The DosR dataset is well-suited to CSDeconv because, in comparison to most mammalian transcription factors, DosR binds only to a small number of sites, allowing the sites to be studied in detail. Moreover, the computational requirements of CSDeconv restrict the number of binding sites that can be analyzed to this scale. Nevertheless, CSDeconv can be applied to mammalian data, and we demonstrate this by analyzing GABP binding over a 2-Mbp segment of human chromosome 19.

In our analysis of DosR binding, we found 24 distinct binding sites distributed over 18 regions, of which 15 regions are upstream of genes whose hypoxic induction has been previously shown to be dependent on DosR [16]. Moreover, our predictions appear spatially accurate with 23 of the 24 predicted sites located within $50 \mathrm{bp}$ of a motif closely resembling that previously identified by Park and co-workers [16]. Notably, four binding sites occur in two closely spaced pairs, and three occur in a closely spaced triplet, and it is clear that these sites cannot be distinguished by using prior peak-calling algorithms. One of the closely spaced pairs occurs in the promoter region of the gene acr (Rv2031c), where the centers of the two distinct sites are separated by only $57 \mathrm{bp}$. That binding occurs at both of these sites was previously established by mobility shift assays [16], and the relative contributions of the two sites to the induction of acr by DosR under hypoxia corresponds qualitatively to the relative binding magnitudes established by our algorithm. In our analysis of GABP binding on chromosome 19, we found 23 distinct binding sites distributed over 15 regions. Of the 23 binding sites, 18 are located within $50 \mathrm{bp}$ of a motif resembling that previously identified $[4,19]$.
Owing to the ability of CSDeconv to call closely spaced binding sites, it is capable of achieving a greater level of accuracy, as determined by motif analysis, than do alternative methods when calling the same number of binding sites. We demonstrate this capability by comparing the performance of CSDeconv with MACS [7] and SISSRs [9], two publicly available ChIP-seq peak finding methods.

\section{Materials and methods \\ Density estimation of enriched regions}

We divided the genome into $N$ nonoverlapping bins. The number of bins $N$ was chosen so that the expected number of reads in each bin, assuming a uniform distribution, would be at least 10 . For simplicity, we rounded bin sizes up to the nearest 100. For the MTB genome, this resulted in 4,412 nonoverlapping bins, each of length 100 $\mathrm{bp}$, and, for the 2-Mbp segment of human chromosome 19 that we studied, this resulted in 182 nonoverlapping bins, each of length 1,100 bp.

We took reads from a ChIP library and reads from a control library and placed them into these bins. We then calculated the log-likelihood ratio (LLR) for independence of the ChIP distribution from the control distribution for each bin, which is given by

$$
\begin{aligned}
& \text { LLR }=n_{\text {ChIP }} \log \left(\frac{n_{\text {ChIP }}}{N_{\text {ChIP }}}\right)+n_{\text {ctrl }} \log \left(\frac{n_{\text {ctrl }}}{N_{\text {ctrl }}}\right)+\left(N_{\text {ChIP }}-n_{\text {ChIP }}\right) \log \left(\frac{N_{\text {ChIP }}-n_{\text {ChIP }}}{N_{\text {ChIP }}}\right) \\
& +\left(N_{\text {ctrl }}-n_{\text {ctrl }}\right) \log \left(\frac{N_{\text {ctrl }}-n_{\text {ctrl }}}{N_{\text {ctrl }}}\right)-\left(n_{\text {ChIP }}+n_{\text {ctrl }}\right) \log \left(\frac{n_{\text {ChIP }}+n_{\text {ctrl }}}{N_{\text {ChIP }}+N_{\text {ctrl }}}\right) \\
& -\left(N_{\text {ChIP }}+N_{\text {ctrl }}-n_{\text {ChIP }}-n_{\text {ctrl }}\right) \log \left(\frac{N_{\text {ChIP }}+N_{\text {ctrl }}-n_{\text {ChIP }}-n_{\text {ctrl }}}{N_{\text {ChIP }}+N_{\text {ctrl }}}\right),
\end{aligned}
$$

where $n_{\text {ChIP }}$ and $n_{\text {ctrl }}$ are the number of ChIP and control reads in the bin, respectively, and $N_{\mathrm{ChIP}}$ and $N_{\mathrm{ctrl}}$ are the total number of ChIP and control reads in the entire dataset, respectively.

We selected those bins with more ChIP reads than control reads whose LLRs exceeded a certain threshold. For each selected bin, we added $300 \mathrm{bp}$ on either side to ensure that the entire enrichment peak is captured, and we call such a genomic region an enriched region. Adjacent or overlapping enriched regions are combined into a single enriched region. Let $k$ be the number of enriched regions.

For each enriched region, we applied kernel density estimation with a gaussian kernel. By following the method of [4], we chose kernel bandwidths empirically to be those that yielded good performance. We chose a bandwidth of 30 for IP reads and a bandwidth of 300 for control reads. For enriched region $i$, we obtain four density functions, $g_{\mathrm{fw}, \mathrm{ChIP}}^{(i)}, g_{\mathrm{rc}, \mathrm{ChIP}}^{(i)} g_{\mathrm{fw}, \mathrm{ctrl}}^{(i)}, g_{\mathrm{rc}, \mathrm{ctrl}}^{(i)}$, for the forward and reverse ChIP reads and the forward and reverse control reads, respectively. We then compute forward and reverse 
enrichment profiles, sampled at integer position values $m$, according to

$$
f_{\mathrm{fw}}^{(i)}[m]:=\frac{g_{\mathrm{fw}, \mathrm{chIP}^{(m)}}^{(i)}}{g_{\mathrm{fw}, \mathrm{ctrl}^{(m)}}^{(m)}}
$$

and

$$
f_{\mathrm{rc}}^{(i)}[m]:=\frac{g_{\mathrm{rc}, \mathrm{chIP}}^{(i)}(m)}{g_{\mathrm{rc}, \mathrm{ctrl}}^{(i)}(m)} .
$$

\section{Initial peak shape estimation}

We make an initial estimate of the shape of an enrichment profile as follows. We aim to select a pulse that is strong (of large amplitude) and narrow, such as to select a pulse that is observed with low noise and that is likely to arise from a single binding site. Thus, for each enriched region, we compute the full width at half maximum (FWHM) and amplitude of the forward and reverse enrichment profiles and compute the average FWHM and amplitude for the region by taking the mean of the forward and reverse values.

We then take the top quartile of the enriched regions according to average amplitude and select the enriched region $i^{*}$ with the smallest average FWHM in this set. In effect, this selects the narrowest peak from among the strongest pulses serving as a good initial estimate of a single binding site. The enriched region $i^{*}$ thus selected is used to compute the initial peak shape.

Specifically, we set the discrete function $h_{0}$ and the scalar $m_{0}$ according to

$\left(h_{\mathrm{o}}, m_{\mathrm{o}}\right):=\underset{h \geq 0,1 \leq m^{\prime} \leq M_{i^{*}}}{\arg \min }\left(\sum_{m=1}^{M_{i^{*}}}\left(f_{\mathrm{fw}}^{\left(i^{*}\right)}[m]-h\left[m-m^{\prime}\right]\right)^{2}+\sum_{m=1}^{M_{i^{*}}}\left(f_{\mathrm{rc}}^{\left(i^{*}\right)}[m]-h\left[m^{\prime}-m\right]\right)^{2}\right)$,

where $M_{i^{*}}$ is the length of the selected enriched region $i^{*}$. The function $h_{0}$ is normalized to a maximal amplitude of unity, and the normalized function describes the initial peak shape.

\section{Iterative blind deconvolution}

We initialize the procedure by setting $h:=h_{0}$. Then, for each enriched region $i$, we solve

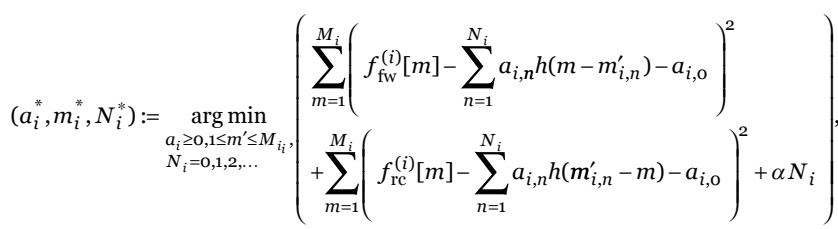

where $M_{i}$ is the length of enriched region $i$, and $\alpha$ is a regularization factor that biases solutions with fewer components. The estimates $a^{*}$ and $m^{*}$ are of the amplitudes and positions of the binding sites, and the estimate $N^{*}$ is of the number of the components in the enriched regions.

We solve the minimization problem for each $i$ by starting with $N_{i}=1$ and solving the minimization over $a_{i}$ and $m_{i}$ by random-restart gradient descent (see, for example, [33]). We then increment $N_{i}$ and solve over $a_{i}$ and $m_{i}$ again, and we continue in this fashion until the objective increases.

For a given $\left(a^{*}, m^{*}, N^{*}\right)$, we reestimate $h$ by assuming that $\left(a^{*}, m^{*}, N^{*}\right)$ are true and estimating the most likely $h$; that is, we solve

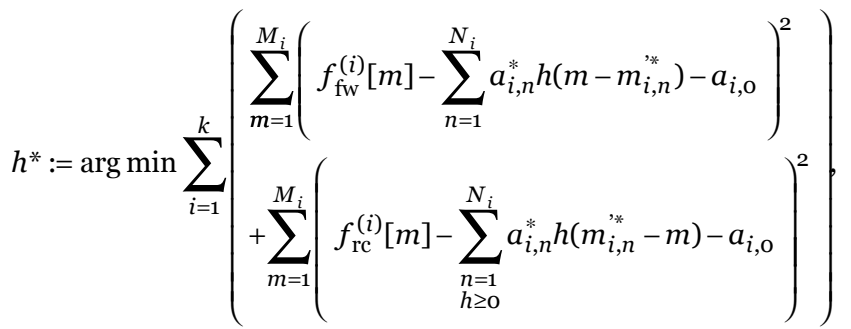

which can be solved as a constrained linear least-squares problem, and set $h:=h^{*}$. We repeat this iterative procedure until convergence in $h$.

\section{DosR ChIP-seq library construction and sequencing}

MTB strains H37Rv and H37Rv: $\Delta$ dosR were grown to early log phase and then exposed to $0.2 \% \mathrm{O}_{2}$ for 2 hours, as described in [22]. The bacilli were fixed by addition of formaldehyde, lysed with bead beating $(6 \times 15$ seconds with cooling on ice between beats), and DNA sheared by sonication. The extract was incubated with anti-DosR antibodies and run over MagnaBind Protein A coated beads (Thermo Fisher Scientific Inc., Rockford, IL, USA). The antibody-bound complex was eluted from the beads, crosslinking was reversed by the addition of SDS and incubation at $65^{\circ} \mathrm{C}$, and DNA fragments were purified by using a QIAquick PCR Purification Kit (QIAGEN Inc., Valencia, CA, USA). The DNA was blunted, and adapters were ligated to each end to facilitate Solexa sequencing. PCR was then used specifically to enrich for DNA fragments with adapter molecules ligated to both ends. DNA obtained from H37Rv was used for the ChIP library, whereas that from H37Rv: $\Delta$ dosR was used for the control library. Sequencing was carried out by using the Illumina/ Solexa Genome Analyzer system, according to the manufacturer's specifications. We obtained a total of $8,361,463$ reads in the ChIP library, of which 5,748,148 (68.7\%) were aligned (some reads were not aligned, as they were not considered uniquely alignable), and a total of $9,627,826$ reads in the control library, of which $6,041,158$ 
$(62.7 \%)$ were aligned. Reads were aligned as described in [3].

\section{GABP ChIP-seq dataset}

ChIP-seq data for the GABP transcription factor in humans was obtained from Valouev and associates [4]. This dataset contains 7,862,231 aligned ChIP reads and $17,404,922$ aligned control reads. We omitted from the dataset all reads that did not lie on chromosome 19 between positions $60,000,000$ and $62,000,000$, which resulted in 27,800 aligned ChIP reads and 19,930 aligned control reads.

\section{Software implementation}

CSDeconv is implemented by using MATLAB R2009a (The Mathworks, Inc., Natick, MA, USA) and is freely available for nonprofit use [34].

\section{Results and Discussion}

An overview of CSDeconv is shown in Figure 1. CSDeconv begins with an initial stage in which enriched regions are identified and kernel density estimation is applied to estimate the probability density functions associated with ChIP and control read locations. For both ChIP and control reads, we estimate probability densities functions for forward reads (reads that align to the forward strand) and reverse reads (reads that align to the reverse strand). To identify enriched regions, we divide the genome into nonoverlapping bins into which reads are binned, and we search for significantly enriched bins by using a log-likelihood ratio (LLR) test. The probability density functions associated with ChIP and control read locations are used to derive enrichment profiles that describe the enrichment level throughout each enriched region for both forward and reverse reads.

From the enrichment profiles, an initial estimate is made of the shape of an enrichment peak. Specifically, we use a heuristic that searches for narrow peaks of large amplitude. This peak shape is used to deconvolve the enrichment profiles (that is, binding site locations and magnitudes are estimated under the assumption that each binding site gives rise to one peak of the given shape). Because the initial peak-shape estimate may be incorrect, the binding-site locations and magnitudes thus obtained are used to reestimate and refine the peak shape. We then return to estimating binding-site locations and magnitudes by using the reestimated peak shape. We repeat this iterative cycle until the change in the peak shape achieved in an iteration is negligible.

\section{Performance}

To test CSDeconv, we applied it to novel ChIP-seq data for the DosR transcription factor in MTB and to existing data for the GABP transcription factor in humans.
DosR is a transcription factor that is believed to play an important role in MTB virulence, and it is therefore important to understand its targets and mechanism of operation. The dosR locus is among the first induced by reduced oxygen [20-22] or low levels of nitric oxide [23], which are conditions thought to reflect in vivo infection. Moreover, DosR is induced rapidly on infection of macrophages $[24,25]$ and mice $[23,26]$. DosR is therefore believed to play an important role in infection, and it is necessary for hypoxic gene induction [16] - a condition used to promote nonreplicating persistence in vitro. Thus, DosR has received significant attention, and a putative motif has been derived for its binding site [16].

GABP is a human transcription factor that was previously studied by using ChIP-seq by Valouev and colleagues [4]. The potential for GABP to bind multiple times in closely spaced regions [27] makes it a suitable test case for blind deconvolution to tease apart multiple binding sites over short distances. As it is currently implemented, CSDeconv cannot be used straightforwardly to analyze genome-wide binding of GABP because the computational requirements of CSDeconv prohibit the analysis of such a large number of enriched regions. CSDeconv can, however, be applied to analyze a subset of all enriched regions, thus demonstrating the efficacy of blind deconvolution, even in the lower sequencing depths that are achieved on mammalian genomes.

To apply CSDeconv effectively, it is necessary to set its parameters to achieve an appropriate level of sensitivity and specificity. Two parameters of principal importance exist: the threshold on the LLR that is used to determine significantly enriched bins, and the regularization factor $\alpha$ that determines the number of binding sites that are called in an enriched region. We determine appropriate levels for these parameters by estimating the false discovery rate (FDR) achieved by various settings. The FDR is estimated by using the same procedure used in a number of ChIPseq and ChIP-chip peak finders $[7,28,29]$ : a sample swap. ChIP and control reads are swapped, CSDeconv is run, and the empirical FDR is calculated as the number of detections in the control (over ChIP) sample divided by the number of detections in the ChIP (over control) sample.

In Figures $2 a$ and $2 b$, we show the empirical FDR for enriched regions as a function of the LLR threshold for the DosR and GABP datasets, respectively. We see that, owing to its lower coverage, larger LLR thresholds are required to achieve low FDRs in the GABP dataset. To ensure that a sufficient number of false enriched regions exist to obtain a good estimate of the FDR for binding sites, we set the LLR threshold to achieve a relatively high empirical FDR for enriched regions. We set the LLR threshold to 18.75 for 


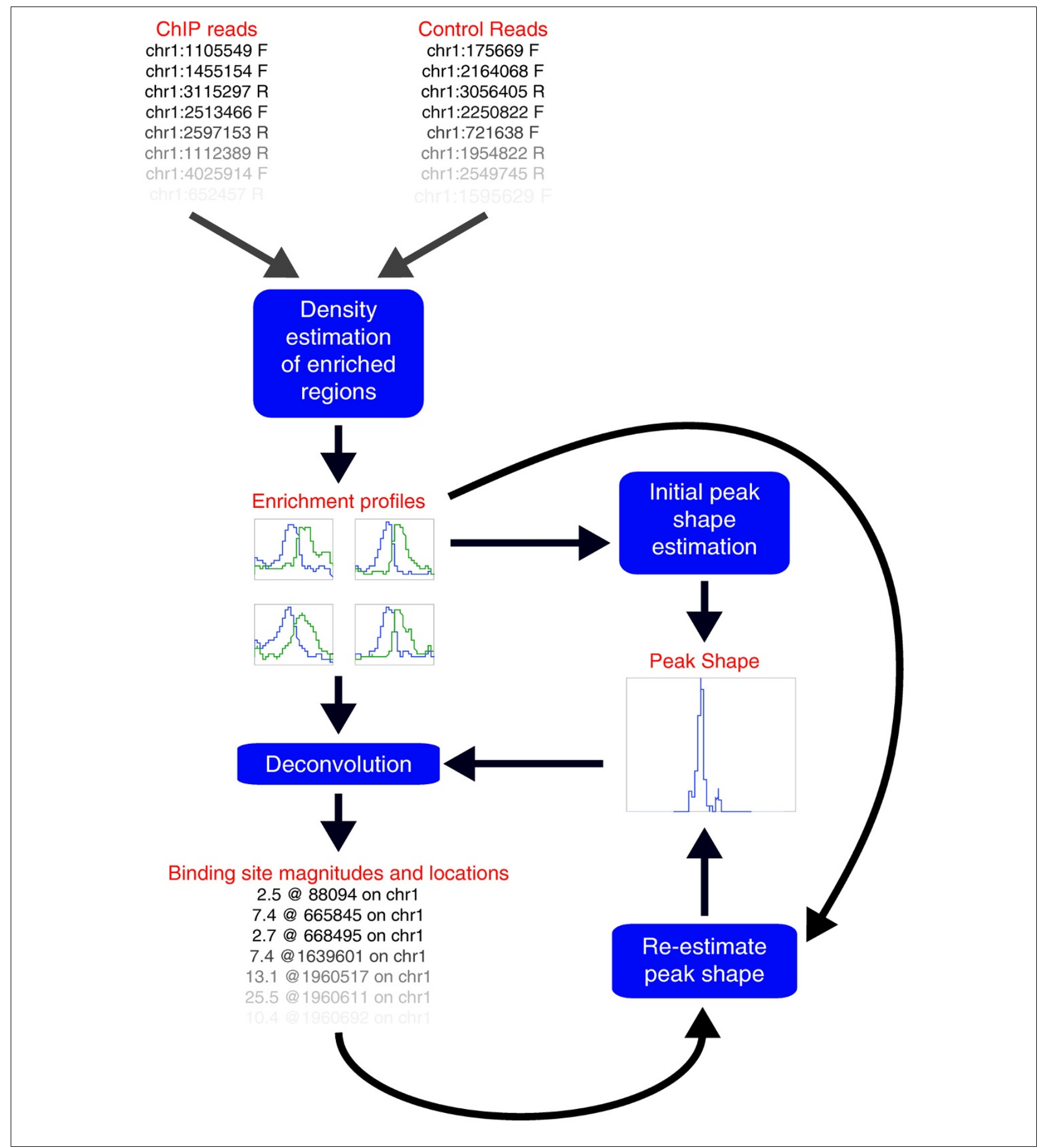

\section{Figure I}

Overview of CSDeconv. After an initial stage in which enriched regions are identified and probability density functions associated with ChIP and control read locations are derived, we obtain enrichment profiles that describe the enrichment level throughout each enriched site for both forward and reverse reads. From the enrichment profiles, an initial estimate is made of the shape of an enrichment peak. The peak shape is used to deconvolve the enrichment profiles, deriving binding-site locations and magnitudes, which are then used to reestimate the peak shape, and this iterative cycle is repeated until convergence. 
the DosR dataset and 38.5 for the GABP dataset, achieving empirical FDRs for enriched regions of 0.389 and 0.40 , respectively.

At these LLR thresholds, we then determine the empirical FDR for binding sites at various levels of $\alpha$. The empirical FDR for binding sites as a function of $\alpha$ is shown in Figures $2 \mathrm{c}$ and $2 \mathrm{~d}$. For the results we report, we set $\alpha$ to 700 for the DosR dataset and to 40,000 for the GABP dataset, achieving low empirical FDRs of 0.042 and 0.044 , respectively.

For DosR, CSDeconv identified a total of 24 binding locations (see Table 1). With MEME [30], we searched for a conserved DNA motif within 50 bp of the binding locations, and we found an 18-bp motif that closely matches the motif previously identified by Park and co-workers [16] from expression analysis (see Figure 3a). Then, by using MAST [31], we searched for the presence of this motif within $50 \mathrm{bp}$ of the binding locations and, for 23 of the 24 binding locations, we found a matching sequence. The average difference of the position estimated by CSDeconv and the center of the motif-matching sequence is $13.9 \mathrm{bp}$, and the average absolute difference is $20.1 \mathrm{bp}$. An examination of the sequences in the 18 enriched regions in which these 24 binding sites occurred did not reveal any likely binding sites that were not called. (a)

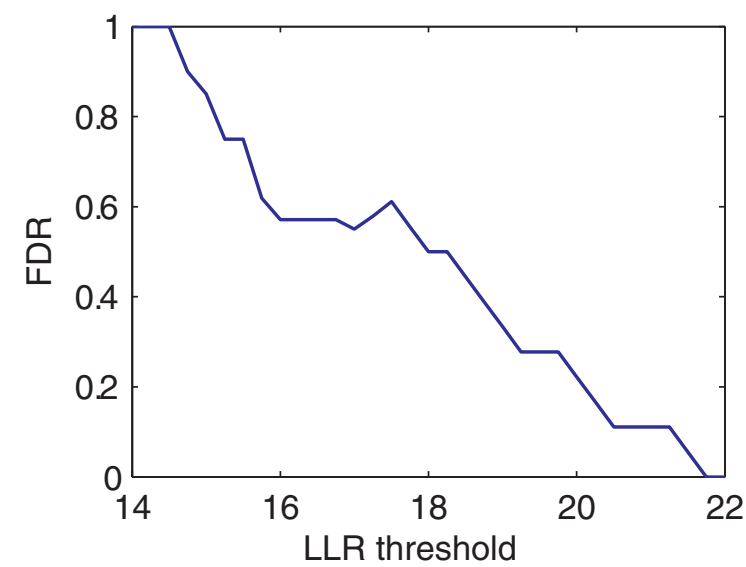

(c)

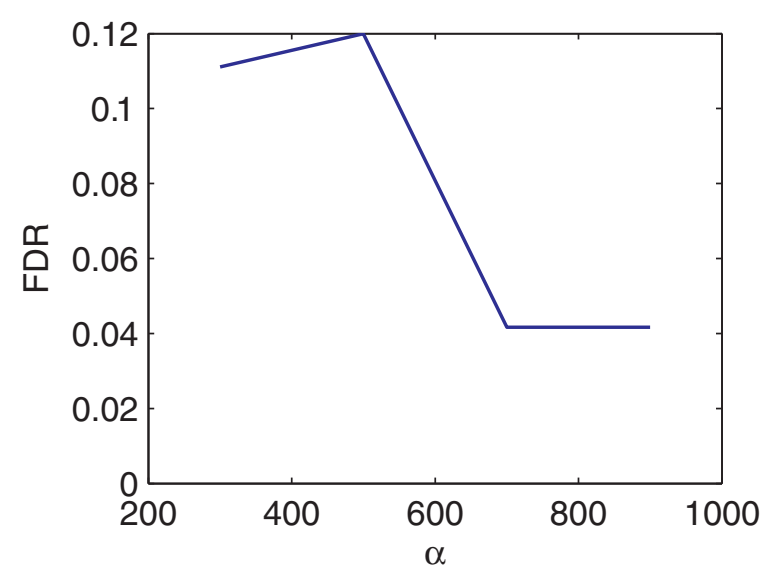

(b)

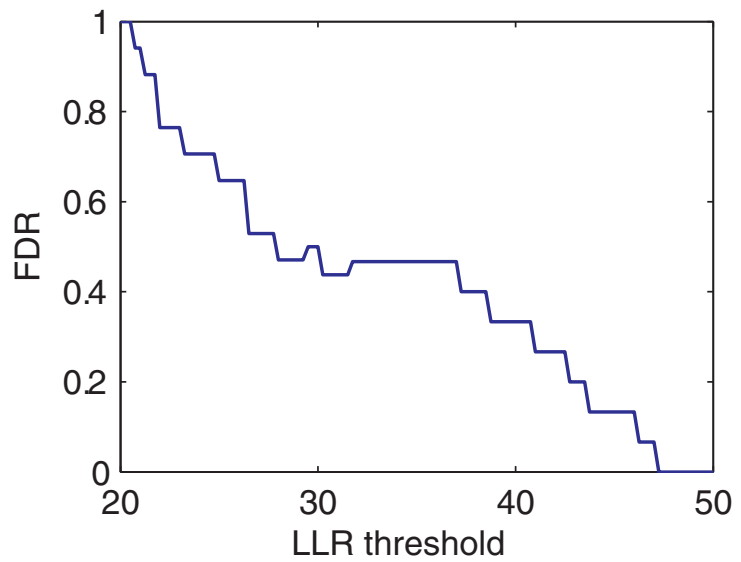

(d)

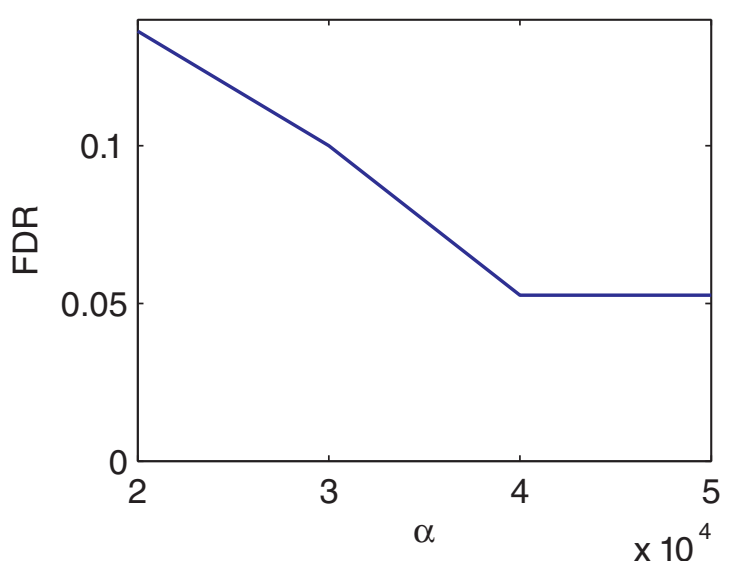

Figure 2

Empirical FDR of CSDeconv. (a, b) The empirical FDR for enriched regions as a function of LLR threshold is shown for the (a) DosR and (b) GABP datasets. (c, d) With the LLR threshold fixed, the empirical FDR for binding sites as a function of the regularization factor $\alpha$ is shown for the (c) DosR dataset (LLR threshold at I8.75) and the (d) GABP dataset (LLR threshold at 38.5). 
Table I

Results of CSDeconv on DosR data

\begin{tabular}{|c|c|c|c|c|c|c|}
\hline Peak ID & Position & Amplitude & Position of motif match & Difference & Absolute difference & Location \\
\hline 1 & $88,097.4$ & 2.4 & $88,125.5$ & 28.1 & 28.1 & Upstream of Rv0079 \\
\hline 2 & $434,899.0$ & 2.8 & $434,926.5$ & 27.5 & 27.5 & In Rv0356c; upstream of Rv0355c \\
\hline 3 & $665,844.1$ & 7.8 & $665,861.5$ & 17.4 & 17.4 & In Rv0573c; upstream of Rv0572c \\
\hline 4 & $668,490.6$ & 2.8 & $668,497.5$ & 6.9 & 6.9 & Upstream of Rv0574c \\
\hline 5 & $801,443.4$ & 3.6 & $801,480.5$ & 37.1 & 37.1 & In Rv0702 \\
\hline 6 & $1,639,602.3$ & 8.5 & $\mathrm{I}, 639,627.5$ & 25.2 & 25.2 & In Rvl453 \\
\hline 7 & $1,960,515.3$ & 13.7 & $1,960,520.5$ & 5.2 & 5.2 & Upstream of Rv1733c \\
\hline 8 & $1,960,611.5$ & 28.0 & $1,960,624.5$ & 13 & 13 & Upstream of Rv1733c \\
\hline 9 & $1,960,697.2$ & 11.0 & & & & Upstream of Rv1733c \\
\hline 10 & $1,965,459.6$ & 10.8 & I,965,47I.5 & 11.9 & 11.9 & Upstream of Rv1737c \\
\hline 11 & $1,965,540.8$ & 15.2 & $1,965,511.5$ & -29.3 & 29.3 & Upstream of Rv1737c \\
\hline 12 & $2,056,358.2$ & 2.7 & $2,056,375.5$ & 17.3 & 17.3 & Upstream of Rv|8|3c, Rv18I4 \\
\hline 13 & $2,238,941.0$ & 9.5 & $2,238,938.5$ & -2.5 & 2.5 & Upstream of Rv1996 \\
\hline 14 & $2,256,459.3$ & 13.0 & $2,256,496.5$ & 37.2 & 37.2 & Upstream of Rv2007c \\
\hline 15 & $2,278,994.7$ & 43.5 & $2,279,005.5$ & 10.8 & 10.8 & Upstream of Rv203 Ic, Rv2032 \\
\hline 16 & $2,279,049.0$ & 25.8 & $2,279,062.5$ & 13.5 & 13.5 & Upstream of Rv203 Ic, Rv2032 \\
\hline 17 & $2,949,475.3$ & 5.7 & $2,949,472.5$ & -2.8 & 2.8 & Upstream of Rv2623 \\
\hline 18 & $2,953,044.8$ & 8.3 & $2,953,074.5$ & 29.7 & 29.7 & Upstream of Rv2626c \\
\hline 19 & $2,954,750.4$ & 5.1 & $2,954,792.5$ & 42.1 & 42.1 & Upstream of Rv2627c, Rv2628 \\
\hline 20 & $2,955,065.5$ & 9.9 & $2,955,031.5$ & -34 & 34 & Upstream of Rv2627c, Rv2628 \\
\hline 21 & $2,955,479.2$ & 9.5 & $2,955,476.5$ & -2.7 & 2.7 & Upstream of Rv2629 \\
\hline 22 & $3,492,069.6$ & 12.0 & $3,492,092.5$ & 22.9 & 22.9 & In Rv3126c; upstream of Rv3|27 \\
\hline 23 & $3,496,438.8$ & 54.7 & $3,496,45 I .5$ & 12.7 & 12.7 & Upstream of Rv3|30c, Rv313| \\
\hline 24 & $3,500,822.1$ & 3.6 & $3,500,853.5$ & 31.4 & 31.4 & Upstream of Rv3134c \\
\hline
\end{tabular}

CSDeconv identifies a total of 24 binding sites. The position of the sequence matching the motif shown in Figure $3 a$ is given if such a sequence exists within 50 bp of the predicted binding site.

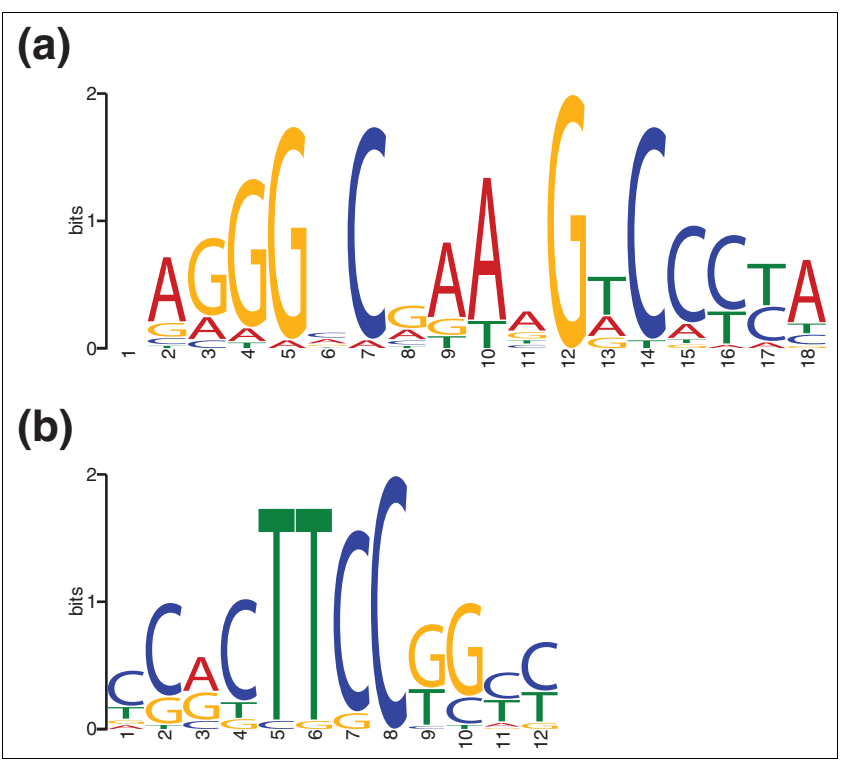

Figure 3

Sequence logos of binding motifs. The sequence logo of the binding motifs found through CSDeconv analysis is shown for (a) DosR and (b) GABP.
Notably, we are able to identify several instances of very closely spaced binding sites. For example, we identify two binding sites upstream of Rv1737c that are separated by only $40 \mathrm{bp}$, and we identify two binding sites upstream of Rv2031c that are separated by only $57 \mathrm{bp}$. As an illustrative example, we show the latter in Figure 4. That binding occurs at both of these sites was previously established by mobility-shift assays [16]. Moreover, our algorithm predicts that more binding occurs at the more upstream of the two sites, which is the site that has been found to be responsible for a greater fraction of the DosR-dependent induction of Rv2031c under hypoxic conditions.

For GABP, we applied CSDeconv to an arbitrarily chosen 2-Mbp segment of human chromosome 19 that starts from chromosome position $60,000,000$. In this segment, we identified 23 GABP-binding locations (see Table 2) of which 17 (74\%) lie within CpG islands, indicative of promoter and control regions [32]. With the same analysis as for DosR, we found a 12-bp motif resembling that previously identified $[4,19]$ that lies within $50 \mathrm{bp}$ of 18 of the 23 binding locations found by CSDeconv (see Figure $3 \mathrm{~b}$ ). The average difference of the position estimated by CSDeconv and the center of the motif-matching sequence is 9.1 $\mathrm{bp}$, and the average absolute difference is $23.5 \mathrm{bp}$. Again, 


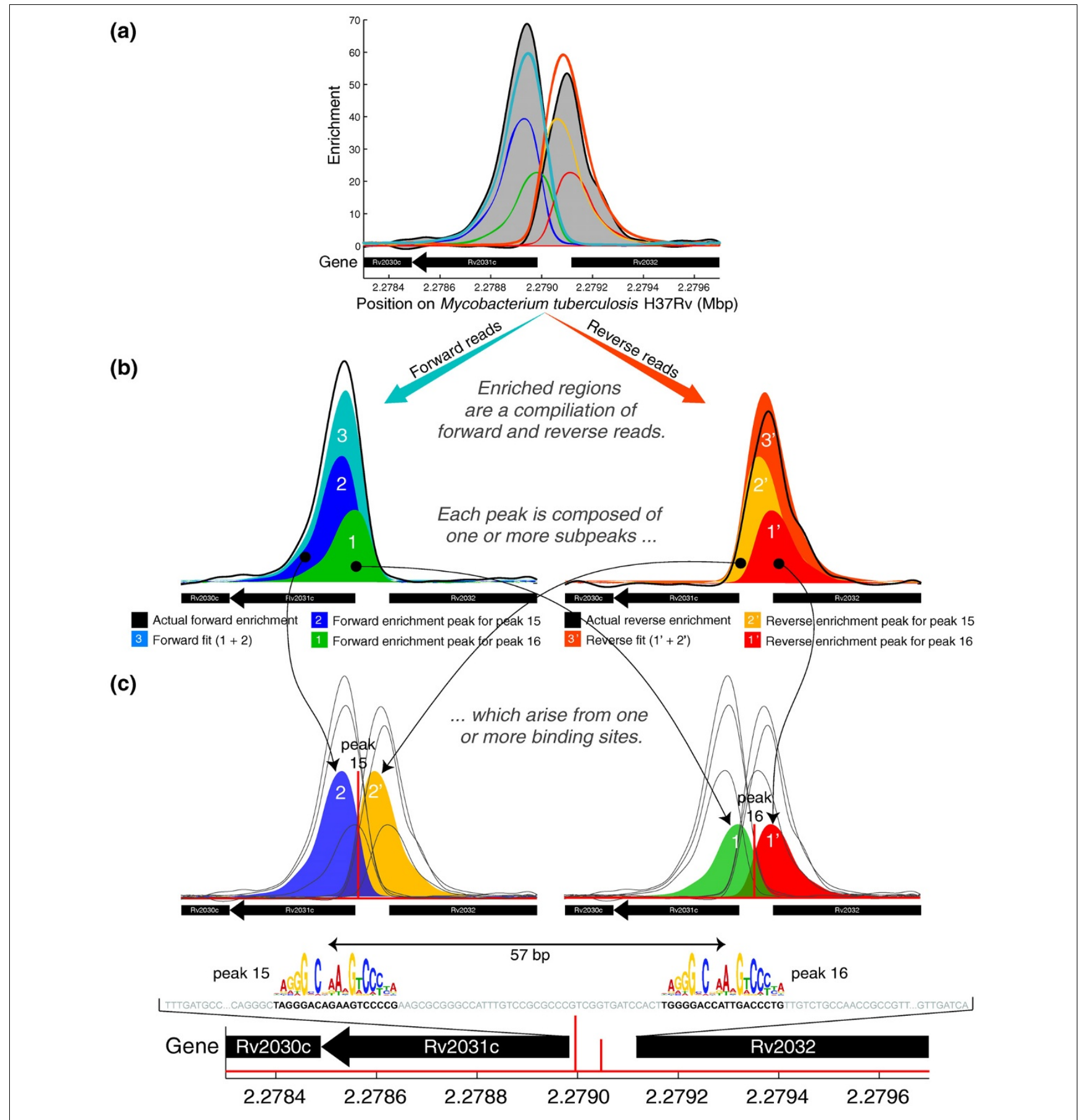

\section{Figure 4}

Illustration of the results obtained by CSDeconv for DosR binding upstream of Rv203 I c. (a) The forward and reverse enrichment profiles obtained after kernel density estimation of the read distributions are shown in black and shaded in gray. Colored lines display various fits arising from estimated binding. Note that no distinct peaks are evident in the enrichment profiles and, in particular, there are no dips. (b) Both forward and reverse reads are associated with fits: the forward fit 3 is the sum of the forward enrichment peaks I and 2, whereas the reverse fit 3' is the sum of the reverse enrichment peaks I' and 2'. (c) The combined forward and reverse enrichment peaks arise from two binding sites, which are peaks 15 and 16 in Table 1 . Motif logos overlay the actual sequence of the intergenic region truncated for brevity, showing the two binding sites, which are separated by a scant $57 \mathrm{bp}$. Enrichment is plotted as the fold magnitude of the ChIP read density over the control read density. 
we identify several instances of very closely spaced binding sites. In particular, we identify two binding sites located at positions $60,209,299.5$ and $60,209,319.5$ that are separated by a mere $20 \mathrm{bp}$.

\section{Comparison with other methods}

Other methods for ChIP-seq data analysis search for peaks of enrichment and call such peaks as single binding sites. They do not deconvolve the peaks into separate binding sites. As such, they are generally incapable of identifying closely spaced binding sites where enrichment peaks overlap and merge into a single peak, as is the case, for example, in Figure 4. We therefore expect that, for the same number of binding sites called, CSDeconv will exhibit a greater level of accuracy than alternative methods, which are based on peak searching. Such alternative methods will miss instances of closely spaced binding sites and instead call false binding sites.

We demonstrate the capabilities of CSDeconv by comparing it with MACS [7] and SISSRs [9], two publicly available ChIP-seq peak-finding methods. For both DosR and GABP, we use MEME and MAST to determine the percent- age of predicted binding sites that have an associated motif within 50 bp for CSDeconv, MACS, and SISSRs, applied at varying levels of stringency.

For the DosR dataset, CSDeconv consistently yields a significantly higher percentage of motif occurrences than do both MACS and SISSRs (see Figure 5). The results we show are obtained with the LLR threshold fixed at 18.75, as before, and we vary $\alpha$ to obtain differing numbers of predicted binding sites. Thus, we expect the accuracy to fall off rapidly after a certain number of predicted sites are called, because the number of enriched regions remains constant. The decline in accuracy is observably noticeable after approximately 25 sites. For the GABP dataset, CSDeconv yields a higher percentage of motif occurrences than MACS and is comparable in performance to SISSRs. The LLR threshold is fixed at 38.5 , as before, so we again expect the accuracy to fall off rapidly for CSDeconv.

Motif occurrence can be used not only to validate binding sites, but potentially also to find them. It may be possible to avoid blind deconvolution by simply searching for multiple, rather than single-motif occurrences around a

Table 2

Results of CSDeconv on GABP data

\begin{tabular}{|c|c|c|c|c|c|c|c|}
\hline Peak ID & Position & Amplitude & $\begin{array}{r}\text { Position of motif } \\
\text { match }\end{array}$ & Difference & Absolute difference & Location & CpG island \\
\hline 1 & $60,209,266.5$ & 145.6 & $60,209,299.5$ & 33 & 33 & Upstream of Hs.63I589 & No \\
\hline 2 & $60,209,352.0$ & 242.4 & $60,209,319.5$ & -32.5 & 32.5 & Upstream of Hs.63I589 & No \\
\hline 3 & $60,209,441.8$ & 32.1 & $60,209,417.5$ & -24.3 & 24.3 & In Hs.63I589 & No \\
\hline 4 & $60,355,290.1$ & 93.1 & $60,355,272.5$ & -17.6 & 17.6 & In TNNI3 & No \\
\hline 5 & $60,355,384.7$ & 109.8 & $60,355,373.5$ & -11.2 & 11.2 & In TNNI3 & No \\
\hline 6 & $60,483,301.5$ & 116.7 & $60,483,320.5$ & 19 & 19 & Upstream of HSPBPI & Yes \\
\hline 7 & $60,508,823.3$ & 53.5 & $60,508,860.5$ & 37.2 & 37.2 & In BRSKI & No \\
\hline 8 & $60,589,079.4$ & 89.0 & $60,589,06 \mid .5$ & -17.9 & 17.9 & $\begin{array}{l}\text { In LOC } 388564, \\
\text { Upstream of RPL28 }\end{array}$ & Yes \\
\hline 9 & $60,784,129.0$ & 152.4 & $60,784,167.5$ & 38.5 & 38.5 & Upstream of ZNF579 & Yes \\
\hline 10 & $60,802,703.2$ & 72.5 & $60,802,723.5$ & 20.3 & 20.3 & In FIZI & Yes \\
\hline II & $60,802,828.5$ & 34.2 & & & & Upstream of FIZI & Yes \\
\hline 12 & $60,808,717.3$ & 19.1 & $60,808,762.5$ & 45.2 & 45.2 & Downstream of ZNF524 & Yes \\
\hline 13 & $60,838,116.9$ & 163.1 & & & & Upstream of ZNF580 & Yes \\
\hline 14 & $60,838,170.7$ & 87.4 & $60,838,194.5$ & 23.8 & 23.8 & Upstream of ZNF580 & Yes \\
\hline 15 & $60,846,738.1$ & 37.1 & & & & Upstream of ZNF58I & Yes \\
\hline 16 & $60,856,978.7$ & 23.4 & & & & Upstream of U2AF2 & Yes \\
\hline 17 & $60,878,268.6$ & 125.4 & $60,878,250.5$ & -18.1 & I8. I & Upstream EPNI & Yes \\
\hline 18 & $60,878,368.7$ & 105.4 & $60,878,386.5$ & 17.8 & 17.8 & Upstream of EPNI & Yes \\
\hline 19 & $61,517,917.1$ & 24.8 & & & & $\begin{array}{l}\text { Upstream of } \\
\text { LOC729994 }\end{array}$ & Yes \\
\hline 20 & $61,518,034.1$ & 98.8 & $61,5 \mid 8,049.5$ & 15.4 & 15.4 & $\begin{array}{l}\text { Upstream of } \\
\text { LOC729994 }\end{array}$ & Yes \\
\hline 21 & $6 I, 518,366.6$ & 26.5 & $61,518,358.5$ & -8.1 & 8.1 & $\begin{array}{l}\text { Upstream of } \\
\text { LOC729994 }\end{array}$ & Yes \\
\hline 22 & $6 I, 74 I, 68 I .4$ & 16.8 & $61,741,681.5$ & 0.1 & 0.1 & In ZFP28 & Yes \\
\hline 23 & $61,741,850.8$ & 25.5 & $61,741,894.5$ & 43.7 & 43.7 & In ZFP28 & Yes \\
\hline
\end{tabular}

CSDeconv identifies a total of 23 binding sites between position $60,000,000$ and $62,000,000$ on chromosome 19. The position of the sequence matching the motif shown in Figure $3 \mathrm{a}$ is given if such a sequence exists within $50 \mathrm{bp}$ of the predicted binding site. 


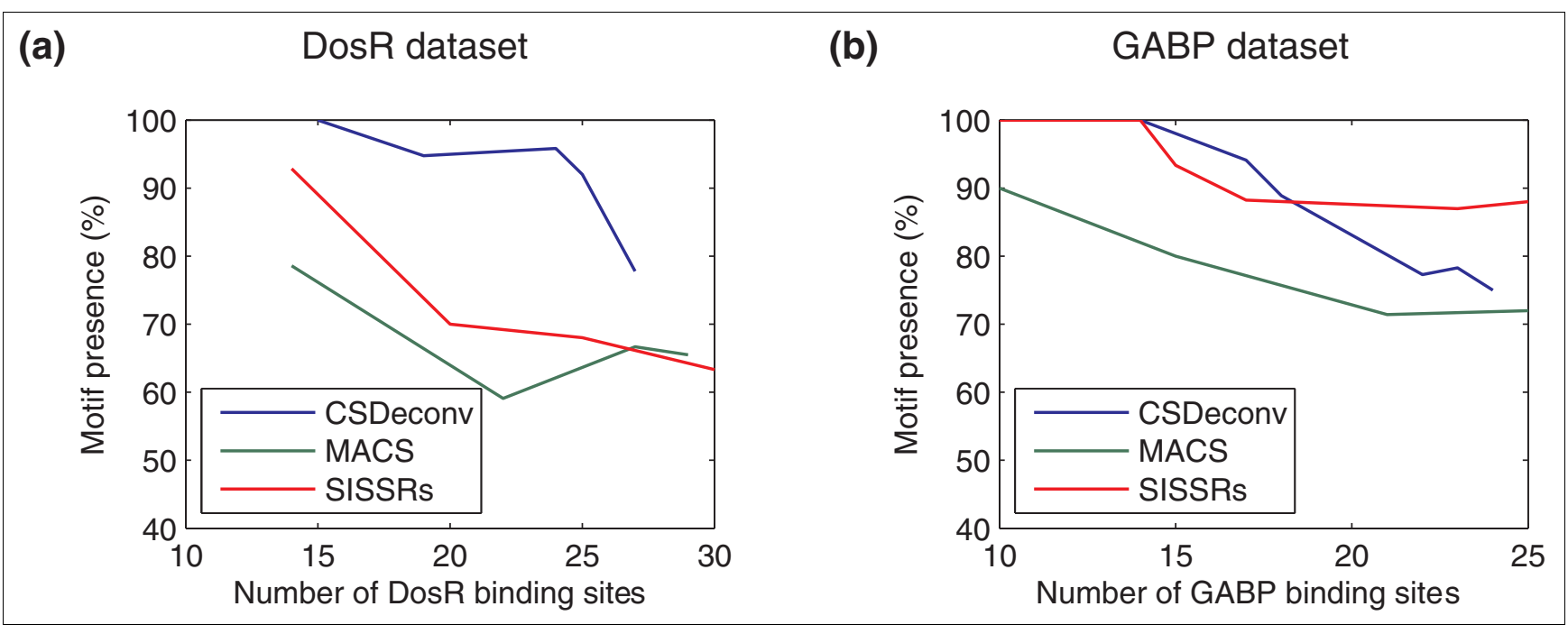

\section{Figure 5}

Comparison of CSDeconv, MACS, and SISSRs by motif analysis. The percentage of predicted binding sites with associated motifs within $50 \mathrm{bp}$ is shown as a function of the number of predicted binding sites with CSDeconv, MACS, and SISSRs for (a) DosR and (b) GABP. For MACS and SISSRs, we take the predicted binding-site location to be the peak center.

ChIP-seq peak. To establish that the performance improvements observed in CSDeconv are due to blind deconvolution and cannot simply be found by motif searching, we compared CSDeconv against a "simplified" version; instead of using blind deconvolution to detect instances of multiple binding sites at a single enriched region, we simply used MEME to search for conserved motifs that can occur arbitrarily many times around peaks in each enriched region. The results of this analysis are shown in Table 3. We see that there are both instances in which binding sites are called by CSDeconv and not be the simplified version and vice versa. In general, the simplified CSDeconv calls more binding sites, and this is especially true in the case of GABP, where the motif is less informative. Cases exist, however, in which the simplified CSDeconv fails to call binding sites that are called by CSDeconv. These cases are supported by read enrichment, and slight modifications to the motif are usually enough to allow a match at those locations, but the simplified CSDeconv has difficulty finding a suitable motif. As for whether the additional binding sites called by the simplified CSDeconv are false positives, this is difficult to determine, as few true negatives are known, especially when it comes to closely spaced binding sites. In the case of the acr (Rv2031c) gene in MTB, however, the binding site in this gene's promoter region that is called by the simplified CSDeconv and is not called by CSDeconv (at position 2279027) is unlikely to be bound by DosR at any significant level, based on previous studies [16]. We conclude, therefore, that the results obtained by CSDeconv cannot simply be obtained by motif searching, and our results indicate that the latter method results in a higher rate of false positives.

\section{Conclusions}

As sequencing becomes faster and cheaper, ChIP-seq will likely become the method of choice for mapping sites of protein-DNA interaction, and methods that can call such sites effectively and accurately from ChIP-seq data will become increasingly important. CSDeconv allows accurate calls to be made in the case of closely spaced transcription factor-binding sites, which is a phenomenon observed frequently, particularly in prokaryotes. The method we use differs substantially from previous techniques in that we use a blind-deconvolution approach, explicitly estimating the shape of an enrichment peak in addition to binding-site locations and magnitudes, thereby distinguishing closely spaced transcription factorbinding sites.

As it is currently implemented, CSDeconv is not attractive for the study of genome-wide binding of transcription factors in mammalian genomes because of its computational requirements. We have, however, demonstrated that CSDeconv can be applied to mammalian ChIP-seq data and is useful for the analysis of such data. Although it is difficult to predict how the number of iterations required by CSDeconv will increase as the number of enriched regions increases, each iteration simply scales linearly. Thus, whereas CSDeconv is currently suited to handle a small number (tens) of enriched regions, it is likely that, with algorithmic improvements, blind deconvolution can 
Table 3

Comparison of CSDeconv with "simplified" CSDeconv

\begin{tabular}{|c|c|c|c|}
\hline \multicolumn{2}{|c|}{ DosR data } & \multicolumn{2}{|c|}{ GABP data } \\
\hline Motif match for CSDeconv & $\begin{array}{r}\text { Motif match for simplified } \\
\text { CSDeconv }\end{array}$ & Motif match for CSDeconv & $\begin{array}{r}\text { Motif match for simplified } \\
\text { CSDeconv }\end{array}$ \\
\hline $88,125.5$ & $88,124.5$ & & $60,209,242$ \\
\hline $434,926.5$ & $434,925.5$ & & $60,209,279$ \\
\hline \multirow[t]{2}{*}{$665,861.5$} & $665,860.5$ & $60,209,300$ & $60,209,299$ \\
\hline & $665,882.5$ & $60,209,320$ & $60,209,319$ \\
\hline $668,497.5$ & $668,498.5$ & & $60,209,356$ \\
\hline $801,480.5$ & & & $60,209,397$ \\
\hline $1,639,628$ & $1,639,629$ & $60,209,418$ & $60,209,417$ \\
\hline \multirow[t]{2}{*}{$1,960,521$} & $1,960,520$ & $60,355,273$ & $60,355,274$ \\
\hline & $1,960,542$ & & $60,355,294$ \\
\hline $1,960,625$ & $1,960,624$ & & $60,355,3 \mid 4$ \\
\hline $1,965,472$ & $1,965,473$ & & $60,355,334$ \\
\hline \multirow[t]{2}{*}{$1,965,512$} & $1,965,513$ & $60,355,374$ & $60,355,375$ \\
\hline & $1,965,533$ & & $60,355,395$ \\
\hline \multirow[t]{3}{*}{$2,056,376$} & $2,056,377$ & & $60,355,415$ \\
\hline & $2,056,410$ & & $60,355,435$ \\
\hline & $2,238,919$ & $60,483,321$ & $60,483,320$ \\
\hline \multirow[t]{2}{*}{$2,238,939$} & $2,238,940$ & & $60,508,762$ \\
\hline & $2,256,475$ & $60,508,861$ & $60,508,862$ \\
\hline $2,256,497$ & $2,256,498$ & $60,589,062$ & $60,589,061$ \\
\hline \multirow[t]{2}{*}{$2,279,006$} & $2,279,007$ & & $60,589,119$ \\
\hline & $2,279,027$ & & $60,589,144$ \\
\hline $2,279,063$ & $2,279,062$ & & $60,589,163$ \\
\hline \multirow[t]{2}{*}{$2,949,473$} & $2,949,472$ & & $60,784,040$ \\
\hline & $2,949,496$ & & $60,784,108$ \\
\hline \multirow[t]{2}{*}{$2,953,075$} & $2,953,074$ & $60,784,168$ & $60,784,167$ \\
\hline & $2,953,099$ & & $60,784,201$ \\
\hline $2,954,793$ & & & $60,802,658$ \\
\hline \multirow[t]{2}{*}{$2,955,032$} & $2,955,033$ & & $60,802,702$ \\
\hline & $2,955,099$ & $60,802,724$ & $60,802,725$ \\
\hline $2,955,477$ & & & $60,802,741$ \\
\hline $3,492,093$ & $3,492,094$ & & $60,802,761$ \\
\hline \multirow[t]{2}{*}{$3,496,452$} & $3,496,453$ & & $60,802,785$ \\
\hline & $3,500,832$ & & $60,802,817$ \\
\hline \multirow[t]{21}{*}{$3,500,854$} & $3,500,853$ & & $60,808,690$ \\
\hline & & & $60,808,717$ \\
\hline & & $60,808,763$ & \\
\hline & & & $60,808,770$ \\
\hline & & & $60,808,789$ \\
\hline & & & $60,808,815$ \\
\hline & & $60,838,195$ & $60,838,196$ \\
\hline & & & $60,846,664$ \\
\hline & & & $60,846,794$ \\
\hline & & & $60,857,070$ \\
\hline & & $60,878,251$ & $60,878,250$ \\
\hline & & & $60,878,292$ \\
\hline & & & $60,878,366$ \\
\hline & & $60,878,387$ & $60,878,388$ \\
\hline & & & $61,517,979$ \\
\hline & & $61,518,050$ & $61,518,051$ \\
\hline & & $61,518,359$ & \\
\hline & & $61,741,682$ & \\
\hline & & & $61,741,862$ \\
\hline & & $61,741,895$ & \\
\hline & & & $61,741,918$ \\
\hline
\end{tabular}

Locations of motif matches for CSDeconv and a simplified CSDeconv that uses motif finding instead of blind deconvolution are listed for both the DosR and GABP datasets. Offsets are observed in the matching sequences for the two methods owing to differing motifs found by the methods. 
be straightforwardly applied to study genome-wide protein-DNA interaction in mammals and other eukaryotes.

\section{Abbreviations}

bp: base pair(s); ChIP: chromatin immunoprecipitation ChIP-Seq: chromatin immunoprecipitation coupled with massively parallel sequencing; DosR: dormancy survival regulator; FDR: false discovery rate; FWHM: full width at half maximum; GABP: growth-associated binding protein; LLR: log-likelihood ratio; MTB: Mycobacterium tuberculosis.

\section{Authors' contributions}

DSL and JEG conceived the project. DSL designed and implemented the algorithm. DSL and BW performed the experiments and wrote the article. AS and DRS generated the DosR dataset.

\section{Acknowledgements}

The authors thank Mark Borowsky, Daniel Neafsey, Toshiro Ohsumi, and Daniel Schwartz for helpful comments and suggestions.

\section{References}

I. Mardis ER: ChIP-seq: welcome to the new frontier. Nat Methods 2007, 4:6|3-6/4

2. Robertson G, Hirst M, Bainbridge M, Bilenky M, Zhao Y, Zeng T, Euskirchen G, Bernier B, Varhol R, Delaney A, Thiessen N, Griffith OL, He A, Marra M, Snyder M, Jones S: Genome-wide profiles of STATI DNA association using chromatin immunoprecipitation and massively parallel sequencing. Nat Methods 2007 , 4:65I-657

3. Mikkelsen TS, Ku M, Jaffe DB, Issac B, Lieberman E, Giannoukos G, Alvarez P, Brockman W, Kim T-K, Koche RP, Lee W, Mendenhall E, O'Donovan A, Presser A, Russ C, Xie X, Meissner A, Wernig M, Jaenisch R, Nusbaum C, Lander ES, Bernstein BE: Genome-wide maps of chromatin state in pluripotent and lineage-committed cells. Nature 2007, 448:553-560.

4. Valouev A, Johnson DS, Sundquist A, Medina C, Anton E, Batzoglou S, Myers RM, Sidow A: Genome-wide analysis of transcription factor binding sites based on ChIP-Seq data. Nat Methods 2008, 5:829-834

5. Johnson DS, Mortazavi A, Myers RM, Wold B: Genome-wide mapping of in vivo protein-DNA interactions. Science 2007, 3 1 6: 1497-1502.

6. Fejes AP, Robertson G, Bilenky M, Varhol R, Bainbridge M, Jones SJM FindPeaks 3.I: a tool for identifying areas of enrichment from massively parallel short-read sequencing technology. Bioinformatics 2008, 24: I729-1730.

7. Zhang Y, Liu T, Meyer C, Eeckhoute J, Johnson D, Bernstein B, Nussbaum C, Myers R, Brown M, Li W, Liu XS: Model-based analysis of ChIP-Seq (MACS). Genome Biol 2008, 9:RI37.

8. Ji H, Jiang $H$, Ma W, Johnson DS, Myers RM, Wong WH: An integrated software system for analyzing ChIP-chip and ChIPseq data. Nat Biotechnol 2008, 26:1293-1300.

9. Jothi R, Cuddapah S, Barski A, Cui K, Zhao K: Genome-wide identification of in vivo protein-DNA binding sites from ChIP-Seq data. Nucleic Acids Res 2008, 36:5221-5231.

10. Kharchenko PV, Tolstorukov MY, Park PJ: Design and analysis of ChIP-seq experiments for DNA-binding proteins. Nat Biotechnol 2008, 26:1351-1359.

I I. Boyle AP, Guinney J, Crawford GE, Furey TS: F-Seq: a feature density estimator for high-throughput sequence tags. Bioinformatics 2008, 24:2537-2538.

12. Zhang ZD, Rozowsky J, Snyder M, Chang J, Gerstein M: Modeling ChIP sequencing in silico with applications. PLoS Comput Biol 2008, 4:el000I58.

13. Rozowsky J, Euskirchen G, Auerbach RK, Zhang ZD, Gibson T, Bjornson R, Carriero N, Snyder M, Gerstein MB: PeakSeq enables sys tematic scoring of ChIP-seq experiments relative to controls. Nat Biotechnol 2009, 27:66-75.

14. Tuteja G, White P, Schug J, Kaestner KH: Extracting transcription factor targets from ChIP-Seq data. Nucleic Acids Res 2009, 37:el I3.

15. Bulyk ML, McGuire AM, Masuda N, Church GM: A motif co-occurrence approach for genome-wide prediction of transcription-factor-binding sites in Escherichia coli. Genome Res 2004, | 4:20|-208.

16. Park H-D, Guinn KM, Harrell MI, Liao R, Voskuil MI, Tompa M, Schoolnik GK, Sherman DR: Rv3 I33c/dosR is a transcription factor that mediates the hypoxic response of Mycobacterium tuberculosis. Mol Microbiol 2003, 48:833-843.

17. Bishop CM: Pattern Recognition and Machine Learning. New York: Springer; 2006

18. Hastie T, Tibshirani R, Friedman J: The Elements of Statistical Learning: Data Mining, Inference, and Prediction. New York Springer; 200I.

19. Virbasius JV, Virbasius C-mA, Scarpulla RC: Identity of GABP with NRF-2, a multisubunit activator of cytochrome oxidase expression, reveals a cellular role for an ETS domain activator of viral promoters. Genes Dev 1993, 7:380-392.

20. Boon C, Dick T: Mycobacterium bovis BCG response regulator essential for hypoxic dormancy. J Bacteriol 2002, 1 84:6760-6767.

21. Dasgupta N, Kapur V, Singh KK, Das TK, Sachdeva S, Jyothisri K, Tyagi IS: Characterization of a two-component system, devR-devS, of Mycobacterium tuberculosis. Tuber Lung Dis 2000, 80: |4|-159.

22. Sherman DR, Voskuil M, Schnappinger D, Liao R, Harrell MI, Schoolnik GK: Regulation of the Mycobacterium tuberculosis hypoxic response gene encoding $\alpha$-crystallin. Proc Natl Acad Sci USA 200I, 98:7534-7539.

23. Voskuil MI, Schnappinger D, Visconti KC, Harrell MI, Dolganov GM, Sherman DR, Schoolnik GK: Inhibition of respiration by nitric oxide induces a Mycobacterium tuberculosis dormancy program. J Exp Med 2003, 198:705-7I3.

24. Schnappinger D, Ehrt S, Voskuil MI, Liu Y, Mangan JA, Monahan IM, Dolganov G, Efron B, Butcher PD, Nathan C, Schoolnik GK: Transcriptional adaptation of Mycobacterium tuberculosis within macrophages: insights into the phagosomal environment. J Exp Med 2003, 1 98:693-704.

25. Yuan Y, Crane DD, Simpson RM, Zhu Y, Hickey MJ, Sherman DR, Barry CE III: The I 6-kDa $\alpha$-crystallin (Acr) protein of Mycobacterium tuberculosis is required for growth in macrophages. Proc Natl Acad Sci USA 1998, 95:9578-9583.

26. Shi L, Sohaskey CD, Kana BD, Dawes S, North RJ, Mizrahi V, Gennaro ML: Changes in energy metabolism of Mycobacterium tuberculosis in mouse lung and under in vitro conditions affecting aerobic respiration. Proc Natl Acad Sci USA 2005 , I02:15629-15634.

27. Yang Z-F, Mott S, Rosmarin AG: The Ets transcription factor GABP is required for cell-cycle progression. Nat Cell Biol 2007 9:339-346.

28. Johnson WE, Li W, Meyer CA, Gottardo R, Carroll JS, Brown M, Liu XS: Model-based analysis of tiling-arrays for ChIP-chip. Proc Natl Acad Sci USA 2006, I 03: I 2457-12462.

29. Song JS, Johnson WE, Zhu X, Zhang X, Li W, Manrai AK, Liu JS, Chen $\mathrm{R}$, Liu XS: Model-based analysis of two-color arrays (MA2C). Genome Biol 2007, 8:RI78.

30. Bailey TL, Williams N, Misleh C, Li WW: MEME: discovering and analyzing DNA and protein sequence motifs. Nucleic Acids Res 2006, 34:W369-W373.

31. Bailey TL, Gribskov M: Combining evidence using p-values: application to sequence homology searches. Bioinformatics 1998, 14:48-54.

32. Takai D, Jones PA: Comprehensive analysis of CpG islands in human chromosomes 21 and 22. Proc Natl Acad Sci USA 2002, 99:3740-3745.

33. Bertsekas DP: Nonlinear Programming 2nd edition. Belmont, MA: Athena Scientific; 1999.

34. CSDeconv [http://www.unisa.edu.au/maths/phenomics/csdeconv/] 